\title{
3D Modeling of Urban Facility Using Point Cloud Data for Realization of Smart City
}

\author{
Joon Kyu Park ${ }^{1}$ and Keun Wang Lee $2^{2 *}$ \\ ${ }^{1}$ Department of Civil Engineering, Seoil University, 28, Yongmasan-ro 90-gil, Jungnang-gu, Seoul, Korea \\ ${ }^{2}$ Department of Multimedia Science, Chungwoon University, 113, Sukgol-ro, Nam-gu, Incheon, Korea
}

(Received April 26, 2020; accepted June 19, 2020)

Keywords: 3D laser scanner, accuracy analysis, point cloud, shape matching, registration

Among the structures that make up a smart city, tunnels are recognized as an important facility in the US and Canada as an infrastructure for the construction of an eco-friendly city. Total station surveying takes a long time to acquire data and has the disadvantage that the Global Navigation Satellite System (GNSS) needs to be able to receive satellites in tunnel surveying. Recently, 3D laser scanners have been attracting attention as a new technology to compensate for the disadvantages of a small amount of data and a long measurement time of the total station. Three-dimensional laser scanners can be used in various fields. In civil engineering, research and application methods often use 3D laser scanners. Because tunnel surveying is difficult in a GNSS survey due to site conditions, total station surveying has been mainly used. As total station surveying takes a lot of time to acquire data, the use of 3D laser scanners for tunnel surveying has recently been increasing. However, when using a 3D laser scanner, a large number of reference points must be installed inside the tunnel for registration and geo-referencing of acquired data. As a new technology, a shape matching method has been introduced in the field of scanning sensors, and its use is expected. In this study, a tunnel survey using a 3D laser scanner was performed and its utility was evaluated. Also, the accuracy of the shape registration method for recent registration was analyzed. The scanning data were acquired by configuring a traverse using the total station function and compared with 10 checkpoints already installed for accuracy verification. As a result of the accuracy evaluation by comparison with the checkpoints, the maximum error was within $6 \mathrm{~cm}$ in the N, E, and $\mathrm{H}$ directions, and these results indicate the plane and elevation allowable accuracy of a 1:1000 digital map, suggesting the applicability of the method using the reference point performance and the laser scanning data. By using scan data, not only analysis of the cross section but also continuous analysis of the shape of the scanning section is possible. An additional evaluation of the accuracy of shape matching of the 3D laser scanner data was performed. By using the scanning data acquired five times, geo-referencing of the data acquired at the first station was performed, and then the data obtained were registered by the shape matching method. A maximum of error of $1 \mathrm{~m}$ occurred at the end of the data. This indicates that the accuracy of the shape registration can be degraded if the same shape is repeated, such as in a tunnel. Therefore,

*Corresponding author: e-mail: kwlee@chungwoon.ac.kr

https://doi.org/10.18494/SAM.2020.2911 
in tunnel surveying, it is judged that the construction of the data only by shape matching may lower the accuracy. Further research can improve the accuracy of the shape registration method, which will improve the efficiency of tunnel surveying.

\section{Introduction}

Among the structures that make up a smart city, tunnels are recognized as an important facility in the US and Canada as an infrastructure for the construction of an eco-friendly city. ${ }^{(1)}$ Toronto plans to build utility tunnels below the city to deploy water pipes and power lines, and to use them as transportation routes for garbage and cargo robots. ${ }^{(2)}$ The US is pursuing a smart city strategy that plans to monitor tunnels and other critical infrastructure facilities. The US intends to apply the Internet of Things (IoT) technology to smart cities. The technology of IoT smart cities will provide the state-of-the-art intelligence and flexibility that cities need to use resources more efficiently. The US government will invest about $\$ 41$ trillion over the next 20 years to upgrade its infrastructure to benefit from the IoT, which will transform the quality of life for urban citizens. ${ }^{(3,4)}$ The current use of GPS, total stations, and 3D laser scanners as surveying equipment in tunnel construction sites has been attracting attention as a new technology to compensate for the disadvantages of the small amount of data and long measurement time of the total station. ${ }^{(5-8)}$ The total station scans the laser at the position to be surveyed, measures the distance using the phase difference of the laser reflected to the target, and then calculates the coordinates of the target using the measured distance and the angle at which the laser is shot. ${ }^{(9-13)}$ Total station surveying takes a long time to acquire data, and there is a disadvantage that the Global Navigation Satellite System (GNSS) needs to be able to receive satellites. ${ }^{(14-19)}$ The GNSS is not used for tunnel surveying because satellites cannot be received inside a tunnel. Figure 1 shows a total station and GNSS receiver. ${ }^{(13)}$

Recently, 3D laser scanners have been attracting attention as devices to compensate for the disadvantages of the small amount of data and long measurement time of the total station. ${ }^{(20-24)}$ A 3D light detection and ranging (LiDAR) laser scanner is a device that stores a group of point clouds of 3D coordinate components $(X, Y$, and $Z$ ) by calculating the return time or phase difference of the returning beam by emitting the laser beam to the measurement target. ${ }^{(25-28)}$ Figure 2 shows a point cloud.
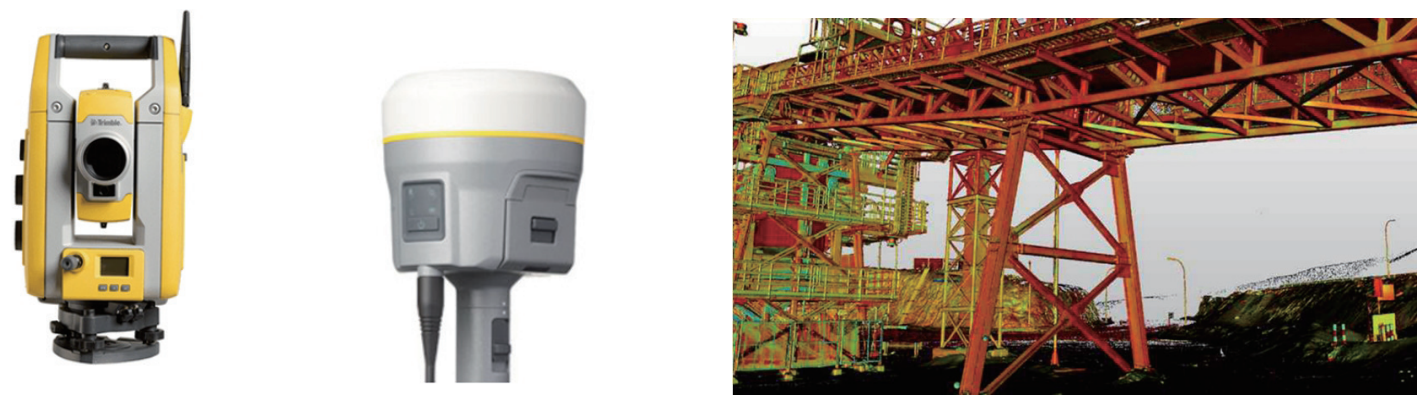

Fig. 1. (Color online) Total station and GNSS receiver.

Fig. 2. (Color online) Point cloud. 
3D laser scanners are used in various fields. In civil engineering, 3D laser scanners are often used in research and application methods. ${ }^{(29-31)}$ Because tunnel surveying is difficult in a GNSS survey due to site conditions, total station surveying has been mainly used. The total station is used to observe the geometry of the construction cross section in 3D coordinates and to compare and analyze the design values at various construction stages. The basic data of the drilling plan to be applied to the subsequent blasting are analyzed using the amount of excavation of the blasting surface, and the excavation error of the tunnel is detected in three dimensions by analyzing the plane position error and the vertical position error of the blasting surface. Figure 3 shows the tunnel excavation work.

After the shotcrete is placed, a 3D laser scanner is used to check the construction thickness of the shotcrete, to determine whether to secure the thickness of the lining concrete to be laid in the future, and to calculate the amount of shotcrete required. ${ }^{(32-36)}$ As total station surveying takes a lot of time to acquire data, the use of 3D laser scanners for tunnel surveying has recently been increasing. However, when using a 3D laser scanner, a large number of reference points must be installed inside the tunnel for registration and geo-referencing of acquired data. As an emerging technology, a shape matching method has been introduced in the field of scanning sensors, and its use is expected. Figure 4 shows SWOT analysis for the application of 3D laser scanners.

In this study, a tunnel survey using a 3D laser scanner was performed and its utility was evaluated. Also, the accuracy of the shape registration method for recent registration was analyzed. Figure 5 shows the flow of this study.

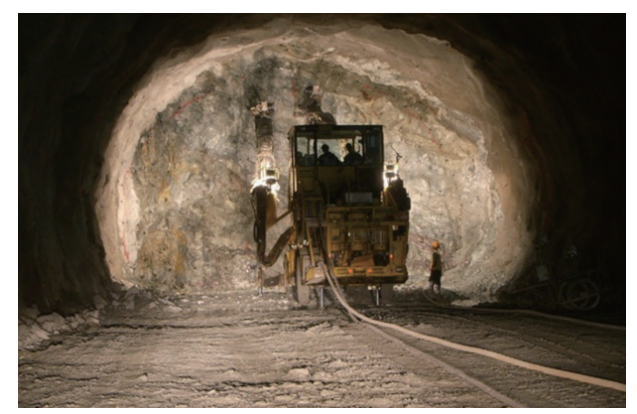

Fig. 3. (Color online) Tunnel excavation work.

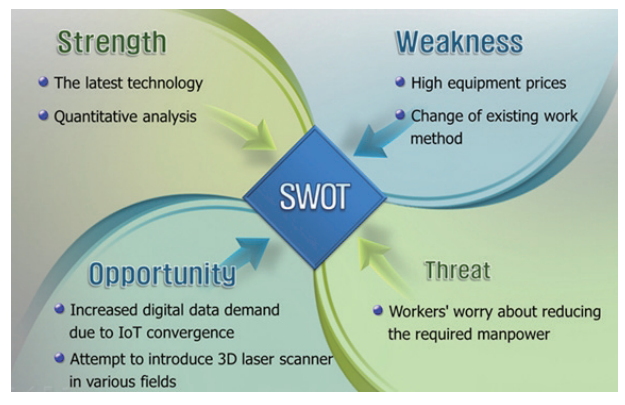

Fig. 4. (Color online) SWOT analysis.

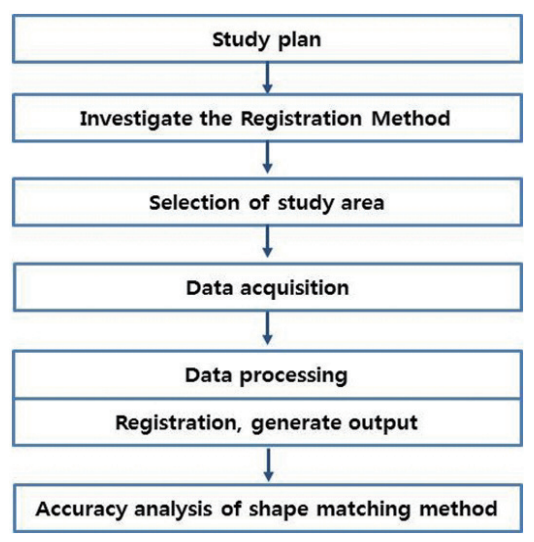

Fig. 5. (Color online) Study flow. 


\section{Point Cloud Registration}

Point cloud registration, also known as point matching in computer vision and pattern recognition, is the process of finding a spatial transform that aligns two sets of points. Finding these transformations involves merging multiple data sets into a globally consistent model and mapping new measurements to known data sets to identify features. In general, the distance measuring method of 3D laser scanning is based on the time of flight (TOF) method, and a basic calculation is as follows. ${ }^{(37)}$

$$
\rho=\frac{1}{2} c \Delta t,\left[\begin{array}{c}
x \\
y \\
z
\end{array}\right]=\left[\begin{array}{c}
\rho \cos \beta \cos \alpha \\
\rho \cos \beta \sin \alpha \\
\rho \sin \alpha
\end{array}\right],
$$

where $\rho$ is the distance, $c$ is the speed of light, and $\Delta t$ is the time difference. The coordinates of a specific point are $x, y$, and $z, \alpha$ is the horizontal angle, and $\beta$ is the vertical angle. The large amount of point data acquired in this way requires registration to be used as geospatial information.

\subsection{Conventional point cloud registration method}

The coordinates of the point cloud acquired through the distance measurement require a registration and geo-referencing process. The existing registration method uses the iterative closest point (ICP) method. The algorithm performs registration in an iterative manner by finding the least squares at the point $\mathrm{S}$ closest to a finite set of points M. The ICP algorithm is given in Fig. 6. ${ }^{(38)}$

This method has the advantage of being intuitive and simple, but has a problem of the need to find the actual coordinate values for each point.

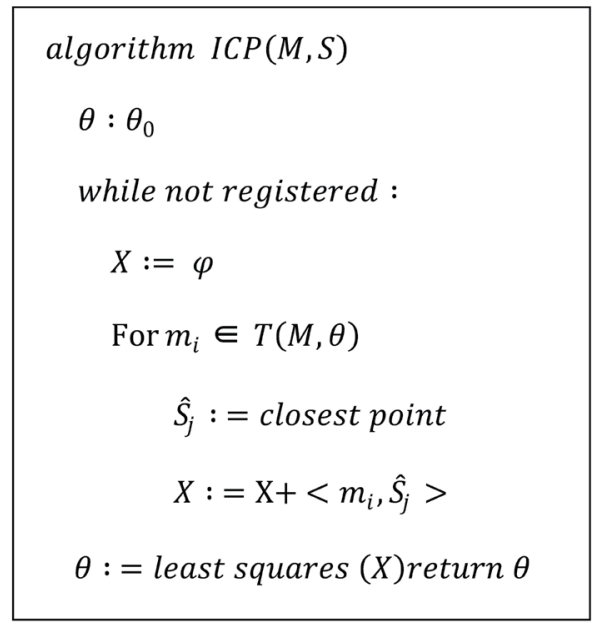

Fig. 6. ICP algorithm. 


\subsection{Point cloud registration method using traverse}

In this study, the traversing method was applied to solve the problem of finding the coordinate values. This is a method of building a control network in the field of surveying. It is also used in geodesy. Traverse surveying networks use surveyed points as a basis for observing the next point after placing survey stations along a track or movement path. The traverse method can acquire coordinates for each point as in Eq. (2). ${ }^{(39)}$

$$
\begin{gathered}
\text { Latitude }=L \cos (A z) \\
\text { Departure }=L \sin (A z)
\end{gathered}
$$

Here, $L$ is the distance and $A z$ is the azimuth. Through this method, the problem of the existing registration method can be addressed. In survey engineering and geodesy, control points (CPs) are often used to establish and observe distances and directions (bearings, angles, azimuths, and elevations). CPs throughout the control network can consist of monuments, benchmarks, vertical controls, and so forth. There are three main types of traverses. Closed traverses start at a station and return to the same station to complete the circuit or run between two known stations. Open traverses do not return to the starting station or close at other known stations. Compound traverses are where the ends of an open traverse are connected to an existing traverse to form a closed traverse. The closed line can be defined by the coordinates at the end point determined by the previous survey.

\subsection{Shape matching registration}

Shape matching registration is a point cloud registration method that has recently begun to attract attention. In the existing method, the registration method is based on the known points, but this method improves the data processing method by extracting information about the shape and registering it on the basis of this information. The basic algorithm of this method is shown in Fig. $7^{(40)}$

In this study, the traverse method and shape matching method are used to overcome the disadvantages of the existing method for point cloud registration. By using these methods to acquire and process data in the field, we tried to evaluate the utility of the new digital technology.

\section{Data Acquisition}

A tunnel construction site in Yangpyeong was selected as the study area for tunnel surveying using a scanning total station, and data on some of the tunnel were acquired. The study area is a site where tunnels are being constructed by private investment and is a suitable area to apply a 3D laser scanner because construction is in progress. Data were obtained for a $300 \mathrm{~m}$ extension of a highway tunnel section. Figure 8 shows the study area and Fig. 9 shows the tunnel construction site. 


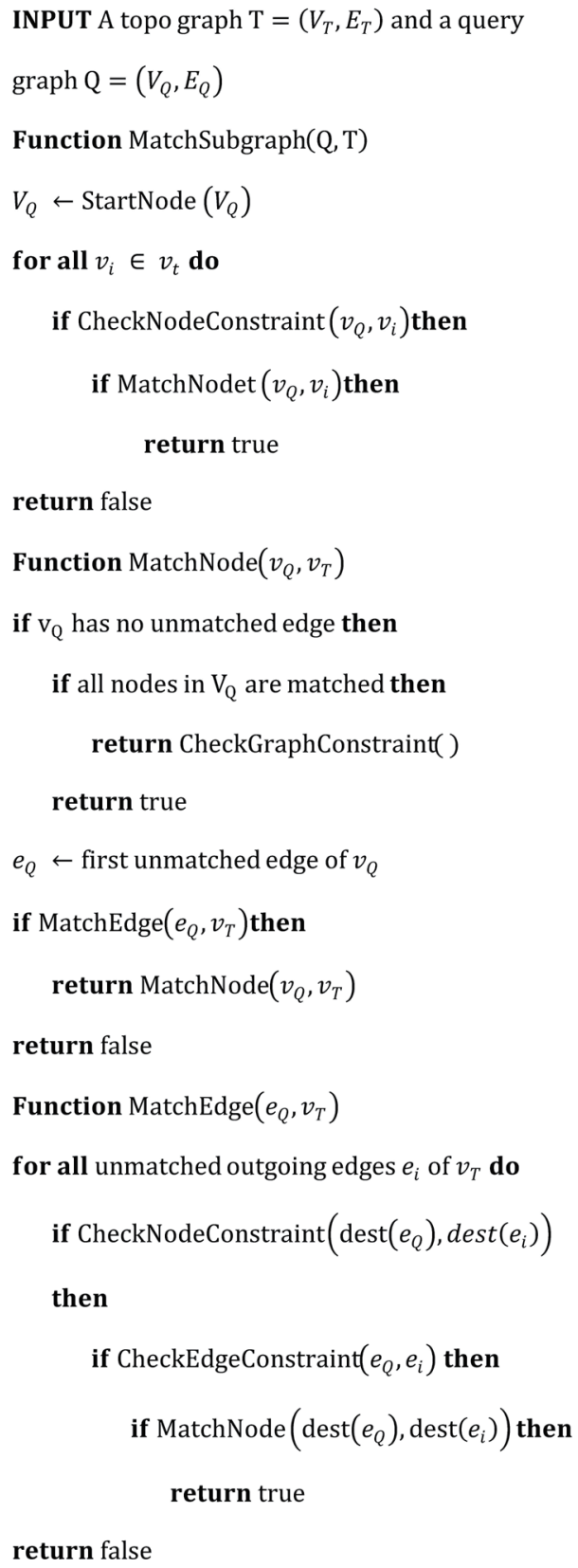

Fig. 7. Shape registration algorithm.

The scanning total station used for data acquisition is the SX10 model, which integrates a total station and a 3D laser scanner. Since the SX10 can apply scanning based on the existing total station surveying method, effective data acquisition is possible. Figure 10 shows the SX10 and the specifications are shown in Table 1.

In addition, the SX10 has a merit that it can acquire data faster than a line scan because a band scan is possible. A band scan is a method of acquiring data by scanning multiple lasers simultaneously. Figure 11 shows the concept of the band scan. 


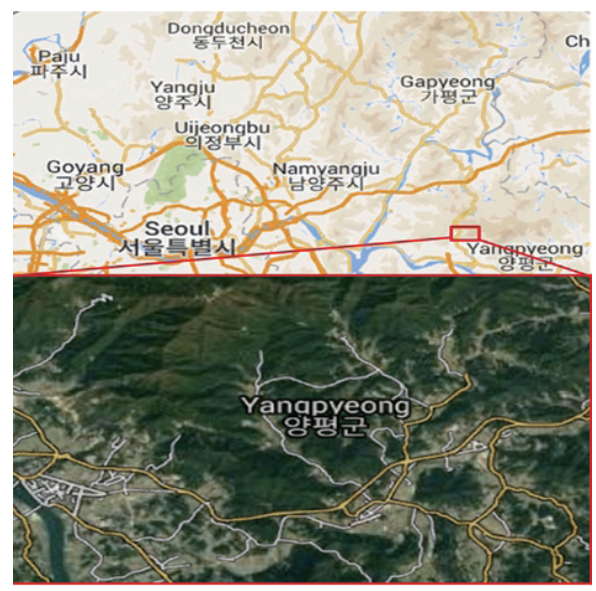

Fig. 8. (Color online) Study area.

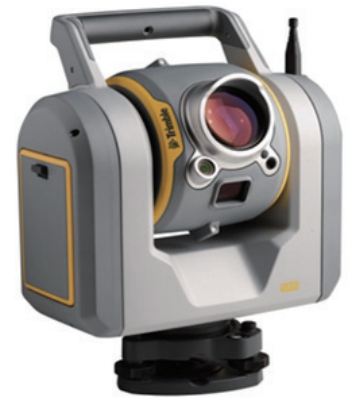

Fig. 10. (Color online) SX10. ${ }^{(13)}$

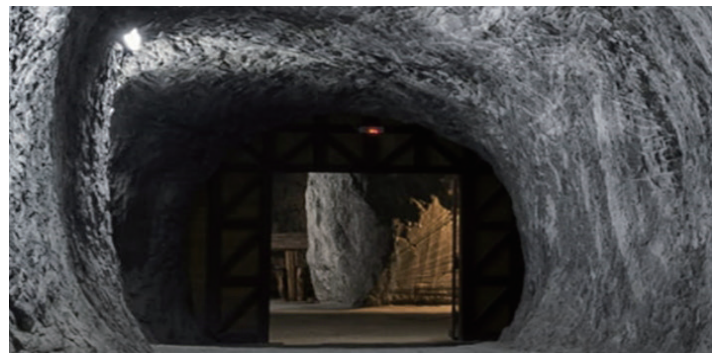

Fig. 9. (Color online) Tunnel construction site.

Table 1

Specifications of SX10. ${ }^{(13)}$

\begin{tabular}{lc}
\hline Item & Description \\
\hline Weight & $7.5 \mathrm{~kg}$ \\
\hline Angle accuracy & 1 " $(0.3 \mathrm{mgon})$ \\
\hline Distance accuracy & prism $: 1 \mathrm{~mm}+1.5 \mathrm{ppm}$ \\
& $\mathrm{DR}: 2 \mathrm{~mm}+1.5 \mathrm{ppm}$ \\
\hline \multirow{2}{*}{ Survey range } & prism $: 5500 \mathrm{~m}$ \\
& $\mathrm{DR}: 800 \mathrm{~m}$ \\
& Autolock $: 300-800 \mathrm{~m}$ \\
\hline Scan speed & 26600 points $/ \mathrm{s}$
\end{tabular}

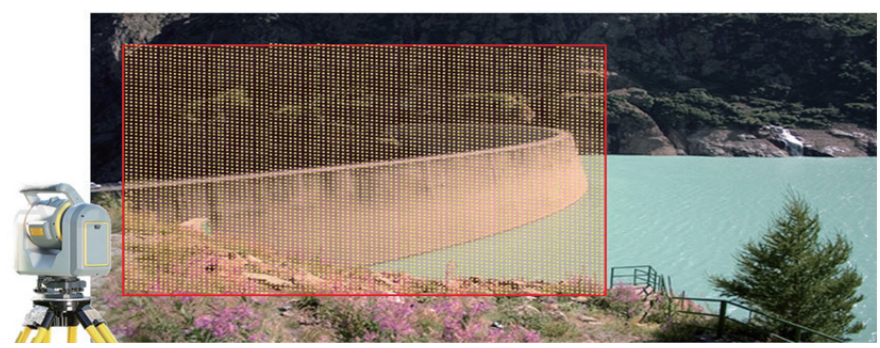

Fig. 11. (Color online) Concept of band scan.

Data acquisition was performed starting from the reference point at the tunnel entrance and moving the mechanical point into the tunnel. In consideration of the redundancy in data processing, scanning was performed once at about $100 \mathrm{~m}$ intervals and data were acquired five times in total with about $50 \%$ redundancy. The time required for data acquisition was shorter than $1 \mathrm{~h} 30 \mathrm{~min}$. Table 2 shows the job status. Figure 12 shows the data acquisition site and Fig. 13 shows the raw data.

The data were acquired by configuring the traverse using the total station function and compared with the 10 checkpoints already installed for accuracy verification. Each checkpoint is a known point and has been surveyed using a total station. Table 3 shows the coordinates of the checkpoints. 
Table 2

Job status.

\begin{tabular}{lc}
\hline Item & Description \\
\hline One measurement time & $12 \mathrm{~min}$ \\
Number of measurements & 4 times \\
Total work time & $1 \mathrm{~h} 30 \mathrm{~min}$ \\
\hline
\end{tabular}

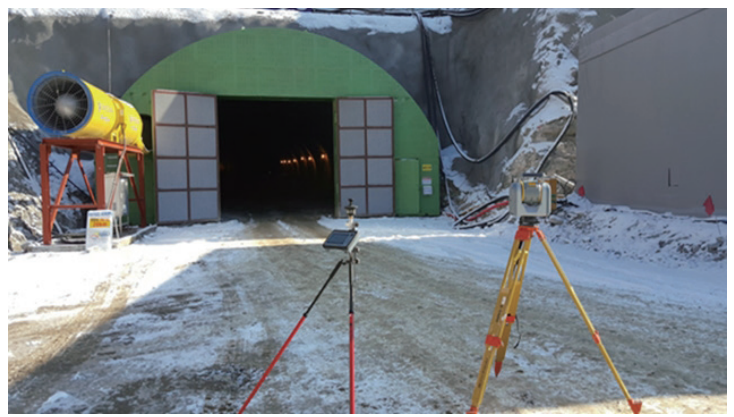

Fig. 12. (Color online) Data acquisition site.
Table 3

Coordinates of checkpoints.

\begin{tabular}{cccc}
\hline No. & $N(\mathrm{~m})$ & $E(\mathrm{~m})$ & $H(\mathrm{~m})$ \\
\hline 1 & 525100.880 & 176957.482 & 572.835 \\
2 & 524962.827 & 177028.469 & 576.010 \\
3 & 525033.949 & 177103.766 & 576.004 \\
4 & 525058.662 & 177115.085 & 575.806 \\
5 & 525011.179 & 177110.816 & 576.090 \\
6 & 524946.974 & 177101.417 & 576.652 \\
7 & 524956.021 & 177042.654 & 576.036 \\
8 & 524887.538 & 176969.374 & 575.820 \\
9 & 525072.828 & 176960.325 & 572.506 \\
10 & 525075.074 & 176930.691 & 572.035 \\
\hline
\end{tabular}

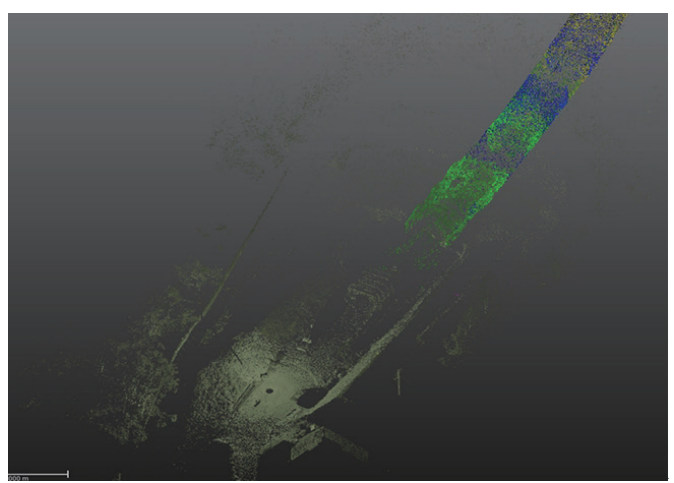

Fig. 13. (Color online) Raw data.

\section{Data Processing and Analysis}

To process the scanning data, data matching was performed with RealWorks software, and a comparison of design sections was performed. Data matching was performed using the traverse function of the scanning total station. Because of the traverse function, the coordinates where the machine is installed are known, so data can be merged automatically without additional registration. Finally, the modeling results for the tunnel were generated for comparison with the design drawings. Figure 14 shows the result of modeling.

To evaluate the accuracy of the tunnel constructed with the 3D laser scanner, the performances of 10 checkpoints were compared. Table 4 and Fig. 15 respectively show a comparison of the accuracies of checkpoints and the result of accuracy analysis.

As a result of the accuracy evaluation compared with the checkpoints, the maximum error was within $6 \mathrm{~cm}$ in the $\mathrm{N}, \mathrm{E}$, and $\mathrm{H}$ directions, and these results indicate the plane and elevation allowable accuracy of a 1:1000 digital map, suggesting the applicability of the method using the reference point performance and the laser scanning data. Table 5 shows the permissible accuracy for numerical mapping given by the Ministry of Land, Infrastructure and Transport, Korea.

Using scan data, not only analysis of the cross section but also continuous analysis of the shape of the scanning section is possible. Models can be created using tunnel design data and 


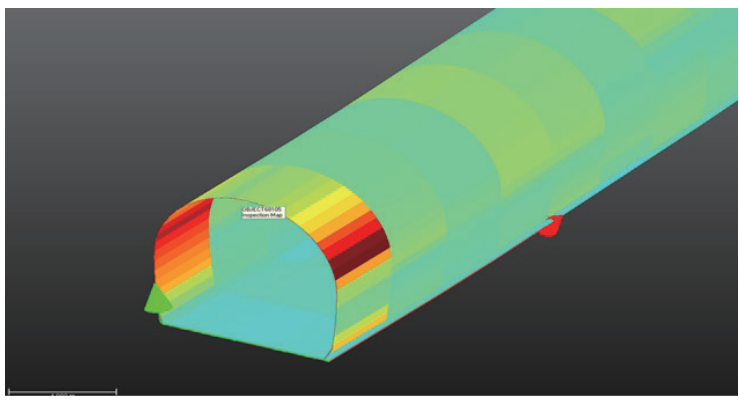

Fig. 14. (Color online) Result of modeling.

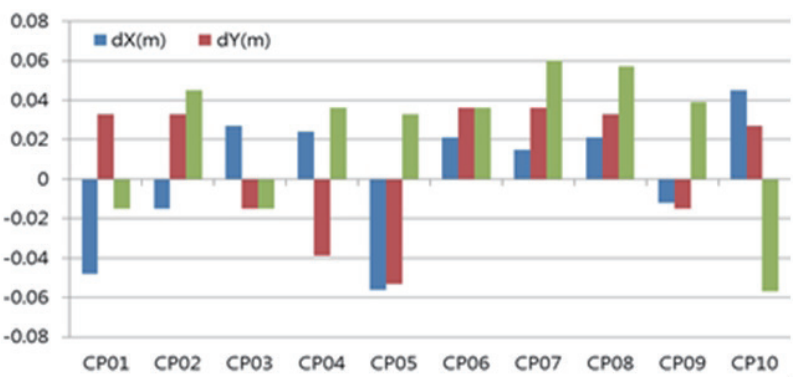

Table 4

Comparison of accuracies of checkpoints.

\begin{tabular}{lrrr}
\hline Point & $d X(\mathrm{~m})$ & $d Y(\mathrm{~m})$ & $d H(\mathrm{~m})$ \\
\hline CP01 & -0.048 & 0.033 & -0.015 \\
CP02 & -0.015 & 0.033 & 0.045 \\
CP03 & 0.027 & -0.015 & -0.015 \\
CP04 & 0.024 & -0.039 & 0.036 \\
CP05 & -0.056 & -0.053 & 0.033 \\
CP06 & 0.021 & 0.036 & 0.036 \\
CP07 & 0.015 & 0.036 & 0.06 \\
CP08 & 0.021 & 0.033 & 0.057 \\
CP09 & -0.012 & -0.015 & 0.039 \\
CP10 & 0.045 & 0.027 & -0.057 \\
\hline
\end{tabular}

Table 5

Permissible accuracy for numerical mapping. ${ }^{(41)}$

\begin{tabular}{ccc}
\hline \multicolumn{3}{c}{ RMSE $(\mathrm{m})$} \\
\hline Plane & Contour & Height \\
\hline 0.2 & 0.3 & 0.15 \\
\hline \multicolumn{3}{c}{ Maximum error $(\mathrm{m})$} \\
\hline Plane & Contour & Height \\
\hline 0.4 & 0.6 & 0.3 \\
\hline
\end{tabular}

Fig. 15. (Color online) Result of accuracy analysis.

then superimposed on the scanning data for analysis. Figure 16 shows the overlap of the design data and the scanning data.

The overlap result in Fig. 16 can be used to generate precise extra-dredging and minimum results at desired intervals along the tunnel section at the correct locations. Figure 17 shows the difference in the lining surface for a specific cross section and Fig. 18 shows the analysis results of the tunnel shape compared with the design data.

The 3D laser scanner deliverables were used to compare the design data with the tunnels and to generate continuous deliveries for the entire work area. The 3D laser scanner can improve the efficiency of tunnel surveying by supplementing the shortcomings of the total station. The advantages of the 3D laser scanner over the conventional total station surveying methods are the fast working speed and the shape of the entire tunnel acquired in the form of a 3D point cloud. The point cloud for the entire tunnel can be analyzed for specific points in the tunnel, and detailed analysis can be performed because of the large amount of data acquired. It is also possible to generate 3D models of excavation, shotcrete, and lining surfaces by acquiring periodic tunnel data. In this study, an additional evaluation of the accuracy of shape matching of 3D laser scanner data was performed. Shape registration can reduce the number of reference point surveys and improve the efficiency of the work. By using the scanning data acquired five times, geo-referencing of the data acquired at the first station was performed, and then the data obtained were registered by the shape matching method. By the shape matching method, the five scanning data were registered in a $300 \mathrm{~m}$ interval without reference point information. Figure 19 shows a comparison between the shape registration result and the traverse method using the reference point. 


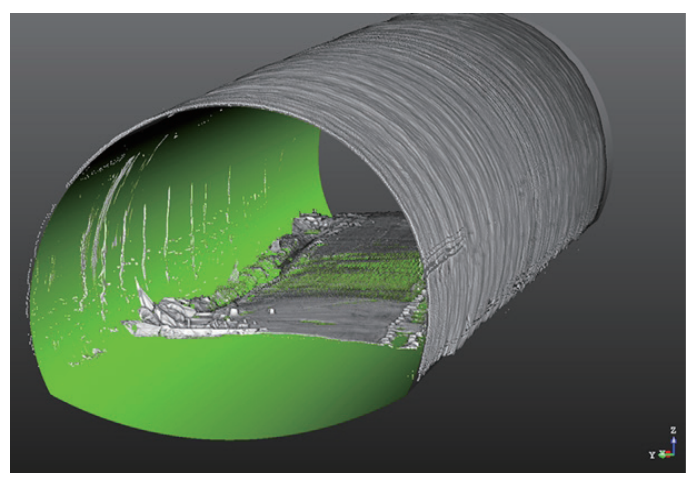

Fig. 16. (Color online) Overlap of the design data and the scanning data.

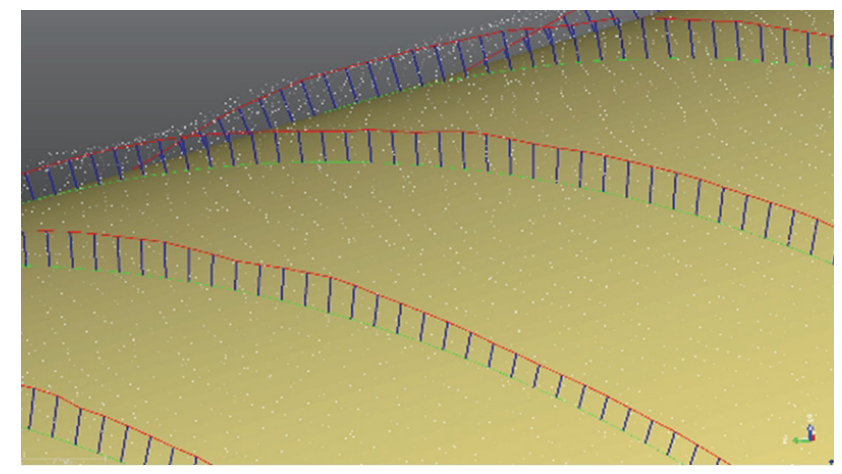

Fig. 17. (Color online) Difference in lining surface for a specific cross section.

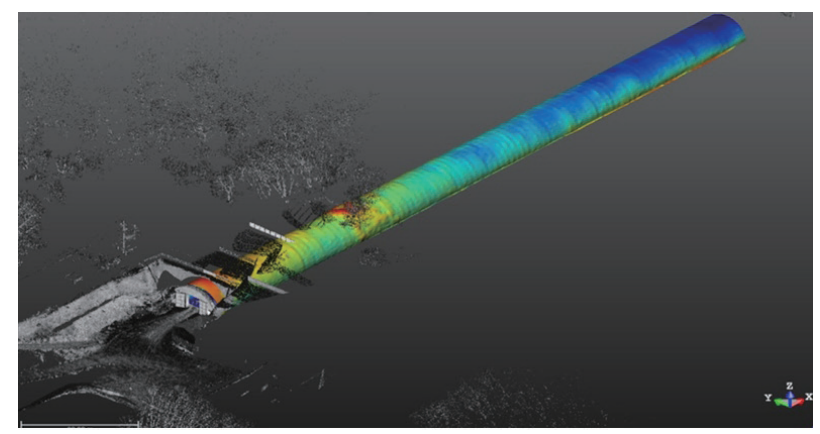

Fig. 18. (Color online) Analysis results of the tunnel shape compared with the design data.

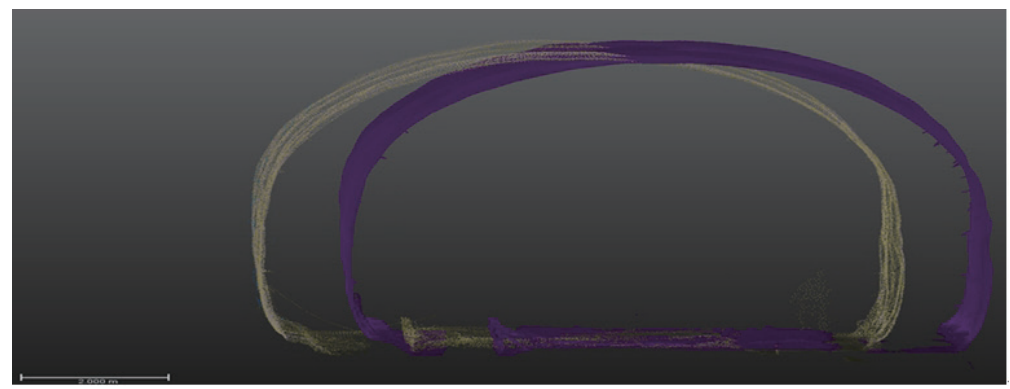

Fig. 19. (Color online) Comparison between the shape registration result and the traverse method.

As shown in Fig. 19, a maximum error of $1 \mathrm{~m}$ occurred at the end of the data. This indicates that the accuracy of the shape registration can be degraded if the same shape is repeated, such as in a tunnel. Therefore, in tunnel surveying, it is judged that the construction of the data only by shape matching may lower the accuracy. The shape registration method can reduce the number of installed targets for registration, thereby improving the efficiency of the work, but the occurrence of errors can be a problem. Through the analysis of the results of the study, the error of shape matching according to the distance was calculated. Table 6 shows the error of shape matching by distance and Fig. 20 shows a graph of the shape matching error. 
Table 6

Error of shape matching by distance.

\begin{tabular}{cc}
\hline Distance $(\mathrm{m})$ & Error $(\mathrm{m})$ \\
\hline 50 & 0.01 \\
100 & 0.34 \\
150 & 0.51 \\
200 & 0.67 \\
250 & 0.84 \\
300 & 1.03 \\
\hline
\end{tabular}

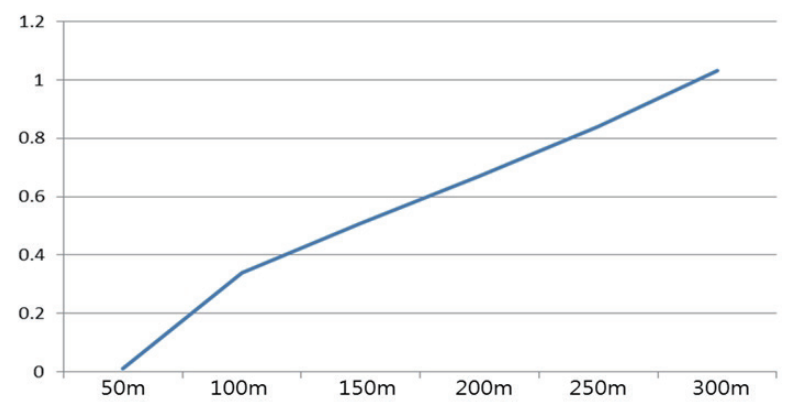

Fig. 20. (Color online) Error of shape matching by distance.

The accuracy of shape registration decreased with increasing distance. Considering the accuracy of the relevant regulations, the shape matching method can be applied within a distance of $100 \mathrm{~m}$. Further research can improve the accuracy of the shape registration method, which will improve the efficiency of tunnel surveying.

\section{Conclusion}

In this study, an accuracy analysis of the new sensing technology of 3D laser scanning was conducted. A tunnel survey using a 3D laser scanner was performed and its utility was evaluated and the accuracy of the shape registration method for recent registration was analyzed. As a result of the accuracy evaluation compared with the checkpoints, the maximum error was within $6 \mathrm{~cm}$ in the N, E, and $\mathrm{H}$ directions, and these results indicate the plane and elevation allowable accuracy of a 1:1000 digital map, suggesting the applicability of the method using the reference point performance and the laser scanning data. Using scan data, not only analysis of the cross section but also continuous analysis of the shape of the scanning section is possible. A maximum error of $1 \mathrm{~m}$ occurred at the end of the data. This indicates that the accuracy of the shape registration can be degraded if the same shape is repeated, such as in a tunnel. Therefore, in tunnel surveying, it is judged that the construction of the data only by shape matching may lower the accuracy. Considering the accuracy of the relevant regulations, the shape matching method can be applied within a distance of $100 \mathrm{~m}$. Further research can improve the accuracy of the shape registration method, which will improve the efficiency of tunnel surveying.

\section{Acknowledgments}

This research was supported by the Basic Science Research Program through the National Research Foundation of Korea (NRF) funded by the Ministry of Science and ICT (No. NRF2018R1C1B6004021). 


\section{References}

1 J. H. So, T. H. Kim, M. J. Kim, J. W. Kang, H. Lee, and J. M. Choi: J. Korean Soc. Transport. 37 (2019) 79. https://doi.org/10.7470/jkst.2019.37.2.079

2 City of Toronto, City Government: https://www.toronto.ca/ (accessed January 2020).

3 Y. Yin, L. Chen, Y. Xu, J. Wan, H. Zhang, and Z. Mai: Mobile Netw. Appl. 25 (2019) 1. https://doi.org/10.1007/ s11036-019-01241-7

4 Smart America, Smart Cities USA: https://smartamerica.org/teams/smart-cities-usa/ (accessed January 2020).

5 S. H. Ju, S. H. Yoon, S. Y. Park, and J. Heo: J. Korean Soc. Surv. Geod. Photogramm. Cartography 36 (2018) 443. https://doi.org/10.7848/ksgpc.2018.36.6.443

6 Y. Yin, S. Aihua, G. Min, Y. Xu, and W. Shuoping: Int. J. Software Eng. Knowl. Eng. 26 (2016) 611. https:// doi.org/10.1142/S0218194016400040

7 S. Y. Kim, J. H. Yu, Y. G. Yu, and H. J. Lee: J. Korean Soc. Surv. Geod. Photogramm. Cartography 34 (2016) 21. https://doi.org/ 10.7848/ksgpc.2016.34.1.21

8 S. Y. Soh, Y. J. Chun, and Ambrose J. M. Itika: J. Korea Academia-Industrial Cooperation Soc. 18 (2018) 438. https://doi.org/10.5762/KAIS.2017.18.4.438

9 Y. Yin, W. Zhang, Y. Xu, H. Zhang, Z. Mai, and L. Yu: IEEE Access (2019). https://doi.org/10.1109/ ACCESS.2019.2914737

10 J. K. Park and M. G. Kim: J. Korea Academia-Industrial Cooperation Soc. 18 (2017) 242. https://doi. org/10.5762/KAIS.2017.18.4.242

11 J. K. Park and K. W. Lee: J. Korea Academia-Industrial Cooperation Soc. 19 (2018) 355. https://doi. org/10.5762/KAIS.2018.19.9.355

12 J. Yu, X. Yang, F. Gao, and D. Tao: IEEE Trans. Cybern. 47 (2017) 4014. https://doi.org/ 10.1109/ TCYB.2016.2591583

13 Trimble Inc.: https://www.trimble.com / (accessed January 2020).

14 M. H. Kim and T. S. Bae: J. Korean Soc. Geod. Photogramm. Cartography 33 (2015) 343. https://doi. org/10.7848/ksgpc.2015.33.5.343

15 T. H. Park, C. M. Jang, S. I. Jang, and B. S. Chun: Trans. Korean Soc. Noise Vib. Eng. 28 (2018) 483. https:// doi.org/10.5050/KSNVE.2018.28.4.483

16 Z. Yu, J. Yu, C. Xiang, J. Fan, and D. Tao: IEEE Trans. Neural Netw. Learn. Syst. 29 (2018) 5947. https://doi. org/10.1109/TNNLS.2018.2817340

17 Y. S. Kang, J. R. Hwang, and K. J. Ahn: Int. J. Control Autom. Syst. 25 (2019) 557. https://doi.org/10.5302/ J.ICROS.2019.19.0015

18 J. L. Zhang, J. F. Yuan, J. Wan, J. Mao, and L. T. Zhu: Concurrency Comput. Pract. Experience 28 (2016) 1751. https://doi.org/10.1002/cpe.3000

19 B. G. Choi, Y. W. Na, and Y. S. Shin: Korean Soc. Surv. Geod. Photogramm. Cartography 35 (2017) 231. https://doi.org/ksgpc.2017.35.4.231

20 H. Si, Z. Chen, W. Zhang, J. Wan, and J. Zhang: Future Generation Comput. Syst. 92 (2019) 1009. https://doi. org/ 10.1016/j.future.2018.07.060

21 C. H. Bae and S. J. Lee: J. Korean Soc. Mech. Eng. 43 (2019) 85. https://doi.org/10.3795/KSME-A.2019.43.2.085

22 S. J. Lee, B. R. Kim, Y. H. Jin, and S. O. Choi: Tunnel Underground Space 27 (2017) 406. https://doi. org/10.7474/TUS.2017.27.6.406

23 C. Jiang, L. Duan, C. Liu, J. Wan, and L. Zhou: VRAA: Cluster Comput. 16 (2013) 639. https://doi.org/10.1007/ s10586-012-0235-6

24 J. K. Park and D. Y. Um: J. Korea Academia-Industrial Cooperation Soc. 19 (2018) 155. https://doi.org/10.5762/ KAIS.2018.19.3.155

25 K. D. Kim, S. H. Jung, K. H. Lee, Y. S. Choi, and M. S. Kim: Korean Soc. Surv. Geod. Photogramm. Cartography 36 (2018) 75. https://doi.org/10.7848/ksgpc.2018.36.2.75

26 K. W. Lee and J. K. Park: J. Korea Acad.-Ind. Cooperation Soc. 19 (2018) 744. https://doi.org/10.15233/ gfz.2018.35.7

27 J. Zhang, J. Xiao, J. Wan, J. Yang, and Y. Ren: Mobile Inf. Syst. (2017). https://doi.org/ 10.1155/2017/3824765

28 J. K. Park and K. W. Lee: J. Korea Academia-Industrial Cooperation Soc. 18 (2017) 638. https://doi. org/10.5762/KAIS.2017.18.12.638

29 D. Qiu: Proc. 2016 Int. Conf. Education, Management, Computer and Society (2016). https://doi.org/10.2991/ emcs-16.2016.433

30 A. R. Kim, D. H. Kim, Y. S. Byun, and S. W. Lee: J. Korean Geotechn. Soc. 34 (2018) 145. https://doi. org $/ 10.7843 / \mathrm{kgs} .2018 .34 .12 .145$ 
31 Y. J. Lee, Y. G. Lee, H. T. Choi, and T. K. Yeo: J. Inst. Electron. Inf. Eng. 56 (2019) 82. https://doi.org/10.5573/ ieie.2019.56.10.82

32 Y. Kim, S. G. Lee, and C. H. Kim: J. Eng. Geol. 27 (2017) 119. https://doi.org/10.9720/kseg.2017.2.119

33 J. S. Park, Y. Lee, and J. H. Kwon: J. Korean Soc. Surv. Geod. Photogramm. Cartography 37 (2019) 101. https://doi.org/10.7848/ksgpc.2019.37.3.101

34 R. L. Sterling: Underground Space 5 (2018) 1. https://doi.org/10.1016/j.undsp.2018.09.001

35 D. G. Lee, E. J. Yoo, and D. C. Lee: J. Korean Soc. Surv. Geod. Photogramm. Cartography 34 (2016) 243. https://doi.org/10.7848/ksgpc.2016.34.3.243

36 J. Zhang, J. Wan, F. Li, J. Mao, and L. Zhuang: Future Generation Comput. Syst. 54 (2016) 490. https://doi. org/10.1016/j.future.2015.03.005

37 J. K. Park, S. Y. Lee, I. T. Yang, and D. M. Kim: J. Korean Soc. Civil Eng. 30 (2010) 191.

38 J. D. Stets, Y. Sun, W. Corning, and S. Greenwald: Proc. SIGGRAPH Asia (2017) 27-30. https://doi.org/ $10.1145 / 3145690.3145729$

39 D. S. Lee and S. E. Hong: J. Korean Soc. Cadastre 6 (2012) 75.

40 H. Tangelder and R.C. Veltkamp: Multimedia Tools Appl. 39 (2004) 145.

41 National Law Information Center, Regulation of Technology Service enterprise: http://www.law.go.kr/ (accessed January 2020).

\section{About the Authors}

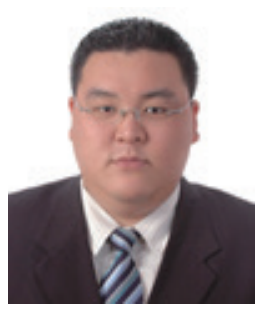

Joon Kyu Park is currently a professor in the Department of Civil Engineering at Seoil University. He received his B.S., M.S. and Ph.D. degrees in civil engineering from Chungnam National University, Korea, in 2001, 2003, and 2008, respectively. His research interests are in the areas of GPS and geo-spatial information engineering. (jkpark@seoil.ac.kr)

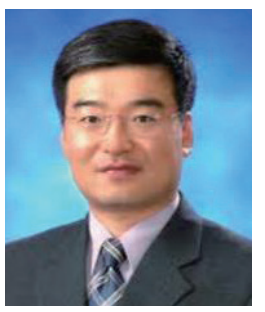

Keun Wang Lee is currently a professor in the Department of Multimedia Science, Chungwoon University. He received his B.S. degree in computer science from Hanbat University in 1993 and his M.S. and Ph.D. degrees in computer science from Soongsil University, Korea, in 1996 and 2000 , respectively. His research interests are in the areas of multimedia communications, multimedia applications, and mobile communications. (kwlee@chungwoon.ac.kr) 